Ciência Florestal, Santa Maria, v. 22, n. 3, p. 541-550, jul.-set., 2012

ISSN 0103-9954

\title{
TÉCNICAS GEOESTATÍSTICAS E INTERPOLADORES ESPACIAIS NA ESTRATIFICAÇÃO DE POVOAMENTOS DE Eucalyptus sp.
}

\author{
GEOSTATISTICAL TECHNIQUES AND SPATIAL INTERPOLATORS IN \\ THE STRATIFICATION OF Eucalyptus sp. STANDS
}

\author{
Isabel Carolina de Lima Guedes ${ }^{1}$ José Márcio de Mello ${ }^{2}$ Carlos Rogério de Mello ${ }^{3}$ \\ Antônio Donizette de Oliveira ${ }^{2}$ Sérgio Teixeira da Silva ${ }^{1}$ José Roberto Soares Scolforo ${ }^{2}$
}

\section{RESUMO}

Os objetivos deste estudo foram verificar a possibilidade do uso da estrutura de dependência espacial para a definição de estratos em povoamentos florestais e comparar, com base no erro de amostragem, a Amostragem Casual Simples (ACS) e a Amostragem Casual Estratificada (ACE), sendo a estratificação realizada por meio dos interpoladores espaciais Inverso do Quadrado da Distância (IQD) e Krigagem. Os dados utilizados foram provenientes de inventários convencionais realizados nos anos de 2006 e 2007, em projetos localizados nos municípios de Curvelo, João Pinheiro e Montes Claros, Minas Gerais. Os resultados mostraram que, dos 50 projetos, $64 \%$ apresentaram grau de dependência espacial de médio a forte, evidenciando que o uso de métodos geoestatísticos possibilita redução no erro sem aumento de custo no inventário. A estratificação com base no interpolador IQD utilizada nos projetos que apresentaram fraco grau de dependência espacial (DE) contribuiu para uma redução em média de 68,4 \% no erro de amostragem. A utilização do interpolador geoestatístico nos projetos que apresentaram de média a forte $\mathrm{DE}$, contribuiu para uma redução média de $47,0 \%$ e $65,7 \%$ no erro de amostragem, respectivamente. Mesmo nos projetos onde a estrutura de dependência espacial se manifestou, a estratificação com base no interpolador IQD gera melhoria das estimativas da ACE em relação à ACS.

Palavras-chave: inventário florestal; dependência espacial; técnicas de amostragem.

\begin{abstract}
The objectives of this study were to verify the possibility of application of the spatial dependence structure for stratus definition in forest stands and to compare, based on the sampling error, Random Sampling Design (ACS) and Stratified Sampling Design (ACE), being the stratification realized by the spatial interpolators Inverse of Square of Distance (IQD) and Kriging. The data was provided from conventional inventories conducted in the years of 2006 and 2007, in clonal eucalypt stands on projects located in Curvelo, João Pinheiro and Montes Claros Counties, in Minas Gerais state. The results showed that, of the 50 projects, $64 \%$ presented medium or high spatial dependence degree, evidencing that geo-statistical methods reduce the error without increasing the costs in the inventory. The stratification based on IQD which was used in the projects with low spatial dependence degree (DE) contributed for a mean reduction of $68.4 \%$ in the sampling error. The use of the kriging in the projects with medium or high DE, contributed for a mean reduction of $47.0 \%$ and $65.7 \%$ in the sampling error, respectively. Even in the projects where the spatial dependence structure was detected, the stratification based on IQD generates improvement of the estimates in the ACE compared to the ACS.
\end{abstract}

Keywords: forest inventory; spatial dependence; sampling techniques.

1. Estudantes do Programa de Pós-graduação em Engenharia Florestal do Departamento de Ciências Florestais, Universidade Federal de Lavras, Caixa Postal 3037, CEP 37200-000, Lavras (MG). isabelcarolinadelima@yahoo. com.br/setesi@uol.com.br

2. Professor do Departamento de Ciências Florestais, Universidade Federal de Lavras, Caixa Postal 3037, CEP 37200-000, Lavras (MG).josemarcio@dcf.ufla.br/donizete@dcf.ufla.br/jscolforo@dcf.ufla.br

3. Professor do Departamento de Engenharia, Universidade Federal de Lavras, Caixa Postal 3037, CEP 37200-000, Lavras (MG).crmello@deg.ufla.br

Recebido para publicação em 20/05/2009 e aceito em 03/06/2011

Ci. Fl., v. 22, n. 3, jul.-set., 2012 


\section{INTRODUÇÃO}

O planejamento e a administração adequada dos recursos florestais se tornam cada vez mais importantes no sentido de se maximizar o aproveitamento dos produtos de base florestal. Existem diversos fatores que influenciam no crescimento das árvores, tais como o clima, o solo, a própria planta, as práticas silviculturais, o melhoramento genético, dentre outros (BOYDEN et al., 2008). Esses fatores sofrem frequentes alterações espaciais e temporais e para garantir o sucesso do planejamento florestal, todos eles devem ser monitorados por meio de técnicas adequadas de amostragem, que sejam eficientes e capazes de gerar informações precisas com menor custo possível.

O inventário florestal, através dos fundamentos da Teoria da Amostragem, estima ou determina uma dada característica de interesse, seja quantitativa ou qualitativa, a partir de uma amostra (PÉLLICO NETTO e BRENA, 1997; MACHADO et al., 2000). O inventário florestal deve gerar informações precisas e a baixo custo sobre a produção da floresta, a fim de subsidiar as tomadas de decisão do manejo florestal (MELLO et al., 2005b).

O processamento dos inventários florestais é realizado utilizando os procedimentos clássicos de estatística, que assumem que as variações espaciais de uma determinada característica são aleatórias, isto é, independentes. Esses procedimentos desconsideram as possíveis relações que possam existir entre as unidades amostrais. Contudo, se a dependência espacial se manifestar, esta deve ser considerada nas análises com o intuito de melhorar a qualidade das estimativas sem que haja aumento nos custos do inventário (MELLO et al., 2005a).

Segundo Cressie (1993), a geoestatística é um ramo da estatística espacial, que controla parte do erro aleatório associado à localização da unidade amostral, isto é, considera que sua localização no espaço exerce influência no comportamento da variância da variável de interesse. O semivariograma é o método geoestatístico que é capaz de diagnosticar a presença da correlação espacial entre as unidades amostradas, e sua modelagem é fundamental para aplicação do interpolador geoestatístico, conhecido como krigagem (ISAAKS e SRIVASTAVA, 1989; MENG et al., 2009).

Uma maneira de controlar a variabilidade existente nos empreendimentos florestais é utilizando a estratificação, que consiste na subdivisão da população total em subpopulações mais homogêneas na característica avaliada. Em geral, os estratos são obtidos com base em informações cadastrais. No entanto, uma estratificação com base na variável de interesse é o ideal (PÉLLICO NETTO e BRENA, 1997; SCOLFORO e MELLO, 2006).

Segundo Kanegae Júnior et al. (2006), a estratificação influencia os custos e a precisão do inventário, em decorrência do controle da variação. Diversos trabalhos abordam a comparação da Amostragem Casual Simples (ACS) com a Amostragem Casual Estratificada (ACE), comprovando que a estratificação reduz o erro do inventário. Mello e Scolforo (2000) compararam diversos procedimentos de amostragem e verificaram que os procedimentos que se basearam na estratificação foram mais precisos dos que os baseados na aleatorização.

Os interpoladores espaciais podem ser divididos em dois grupos: determinísticos e estatísticos. O interpolador determinístico mais utilizado é o Inverso da Distância Ponderada (IDW), que, para a predição de locais não amostrados, utiliza informações de pontos amostrados que circundam o local da predição, isto é, os pontos amostrados vizinhos (LANDIM, 1998; YAMAMOTO, 2001). A krigagem é um interpolador estatístico que estima valores em pontos não amostrados a partir de informações dos pontos amostrados, considerando a estrutura de dependência espacial da característica em estudo (MATHERON, 1963; VIEIRA, 2000). Assim sendo, técnicas de interpolação espacial podem ser aplicadas na pós-estratificação dos povoamentos, pois, com o controle da variabilidade da característica de interesse, é possível estabelecer estratos bem definidos (KANEGAE JÚNIOR et al., 2006).

Diante do exposto, os objetivos deste estudo foram: a) avaliar o potencial de uso dos interpoladores espaciais, na ocorrência de dependência espacial ou não, para definição de estratos; b) comparar o erro de amostragem da Amostragem Casual Simples com a Amostragem Casual Estratificada.

\section{MATERIAL E MÉTODO}

\section{Caracterização da área de estudo}

Este trabalho foi realizado em povoamentos clonais de Eucalyptus sp., pertencentes à empresa Vallorec \& Mannesman Florestal, localizados nos municípios de Curvelo, João Pinheiro e Montes 
Claros, em Minas Gerais.

A área total do estudo compreende $9.796,6$ ha, sendo 3.197,8 ha no município de Curvelo; 3.842,9 ha em João Pinheiro e 2.755,9 ha em Montes Claros. Foram amostrados povoamentos nas idades de $24,36,48$ e 60 meses, nos espaçamentos de 3,00 $\mathrm{m} \times 3,00 \mathrm{~m}$ e $3,00 \mathrm{~m} \times 2,00 \mathrm{~m} \times 6,00 \mathrm{~m}$, trata-se de um espaçamento adotado pela empresa para otimização do processo de colheita.

O clima destes municípios, segundo a classificação de Köppen, é do tipo Aw, que se caracteriza como Tropical úmido de savana, com inverno seco e verão chuvoso e a temperatura média do mês mais frio é superior a $18^{\circ} \mathrm{C}$ (ANTUNES, 1986).

\section{Caracterização da base de dados}

Os dados utilizados neste estudo foram oriundos de inventários convencionais realizados em 2006 e 2007. O procedimento de amostragem adotado foi o Sistemático Desencontrado (COCHRAN, 1977), lançando-se uma parcela circular, com área de $400 \mathrm{~m}^{2}$ a cada 10 hectares de floresta.

Em todas as parcelas mediu-se a circunferência a $1,30 \mathrm{~m}$ do solo (CAP) de todas as árvores, a altura total das dez primeiras árvores centrais, a altura das árvores dominantes, conforme o conceito de Assmann (PRODAN et al., 1997), e o centro de todas as parcelas foi georreferenciado. A estimativa do volume foi realizada por meio de equações volumétricas, associadas às equações hipsométricas. Foram selecionados 50 projetos para a realização deste estudo.

\section{Estudo variográfico}

Primeiramente, foi realizada a análise exploratória dos dados com o intuito de entender o comportamento geral dos mesmos. Embora desconsidere a estrutura de dependência espacial, esta análise permite a identificação de dados discrepantes. Esta primeira etapa se constituiu da determinação de medidas de posição e dispersão, gráficos de tendência em relação à longitude e à latitude e avaliação da normalidade dos dados através do teste de Shapiro Wilk com nível de significância $\alpha=5$ \% (FERREIRA, 2005; SHAPIRO e WILK, 1965).

O estudo variográfico foi realizado com o intuito de verificar a estrutura de dependência espacial, caracterizando o semivariograma experimental de cada projeto. No processo de modelagem do semivariograma, foram ajustados os modelos Esférico e Exponencial para a característica volume, descritos por Journel e Huijbregts (1978), a fim de se obter o conjunto de parâmetros para as respectivas funções. Os ajustes foram feitos pelos Métodos dos Quadrados Mínimos Ordinários e da Máxima Verossimilhança, utilizando o programa $\mathrm{R}$ (R DEVELOPMENT CORE TEAM, 2007), usando o pacote geoR (RIBEIRO JÚNIOR e DIGGLE, 2001).

A avaliação do desempenho de cada modelo se deu pelo Critério de Informação de Akaike (AIC), conforme Webster e McBratney (1989). O AIC foi obtido por meio da função de Máxima Verossimilhança, a partir dos parâmetros ajustados para os modelos. Com os parâmetros do modelo espacial foi determinado o grau de Dependência Espacial (DE), que é uma relação percentual entre a variação estruturada $\left(\sigma^{2}\right)$ em relação ao patamar $\left(\tau^{2}+\sigma^{2}\right)$, apresentado por Biondi et al. (1994), com a seguinte classificação: $\mathrm{DE}>75 \%$, alto grau de dependência espacial; $35 \leq \mathrm{DE} \leq 75 \%$, médio grau de dependência espacial e $<35 \%$, fraca estrutura de dependência espacial.

\section{Estratificação com base nos interpoladores estatístico e determinístico}

Em meio aos projetos avaliados que apresentaram fraca dependência espacial, foram selecionados cinco para a realização da estratificação, por meio do interpolador Inverso da Distância Ponderada (IDW), com expoente 2, sendo, neste caso, conhecido como Inverso do Quadrado da Distância (IQD), descrito por Yamamoto (2001). Entre os projetos que apresentaram grau de dependência espacial de médio a forte, foram selecionados cinco de cada situação para a realização da estratificação por meio do IQD e da krigagem. A estimativa dos pontos não amostrados foi realizada com base krigagem pontual, descrita por Isaaks e Srivastava (1989) e Vieira (2000). A pós-estratificação foi realizada com base na característica volume, para posterior comparação entre a Amostragem Casual Estratificada (ACE) e a Amostragem Casual Simples (ACS).

O número de classes adotado foi igual a quatro, com base nos estudos realizados por Kanegae Júnior et al. (2006), que constataram que esse número se mostra mais operacional e mais eficiente no controle da variabilidade. Contudo, quando não foi possível a obtenção de quatro estratos, este número foi reduzido. 


\section{Processamento do inventário florestal}

Para avaliar o desempenho dos estratificadores, efetuou-se o cálculo do erro de amostragem $(E \%)$ considerando as seguintes situações: adoção de toda a área como um único estrato, com o processamento do inventário realizado através dos estimadores da Amostragem Casual Simples; considerando a estratificação obtida pelos interpoladores espaciais, com o processamento do inventário efetuado pelos estimadores da Amostragem Casual Estratificada. Após a estratificação, definiram-se as parcelas de cada estrato e, os processamentos da ACE foram comparados àqueles da ACS. A formulação detalhada desses dois procedimentos pode ser encontrada em Cochran (1977), Péllico Netto e Brena (1997) e Scolforo e Mello (2006).

\section{RESULTADOS E DISCUSSÕES}

Pelo teste de Shapiro Wilk, observou-se que $90 \%$ dos projetos avaliados apresentaram normalidade, sendo esta uma tendência da variável dendrométrica volume em plantios de eucalipto, conforme constatado nos trabalhos de Assis (2005); Kanegae Júnior et al. (2007); Mello et al. (2005a); Mello et al. (2009). Apesar da normalidade dos dados não ser uma exigência para aplicação das técnicas geoestatísticas, esta distribuição atribui às inferências outras propriedades estatísticas ótimas, como a Máxima Verossimilhança (DIGGLE e RIBEIRO JÚNIOR, 2007).

Na Tabela 1 é possível observar o valor médio de volume por hectare e o coeficiente de variação médio em cadaidadedentrodas respectivas regiões avaliadas. São informações úteis para uma primeira inferência sobre a produtividade por idade em três regiões de importância para o setor florestal mineiro, especialmente para definição da intensidade amostral em inventários. O coeficiente de variação variou de 11 até aproximadamente 35 $\%$, dependendo da idade e da região.

Em todos os projetos verificou-se a inexistência de tendência da variável volume em relação à latitude e à longitude. Esta situação foi observada em todos os projetos avaliados. Esta é uma condição importante, pois na presença de tendência não há estacionaridade, o que comprometeria a Hipótese Estacionária de $1^{\underline{a}}$ Ordem, inviabilizando a aplicação das técnicas geoestatísticas (DIGGLE e RIBEIRO JÚNIOR, 2007). A Figura 1 mostra a relação do volume com as coordenadas UTM. Resultados semelhantes, com relação à ausência de tendência da característica volume foram detectados por Mello (2005a), Mello et al. (2009) e Kanegae Júnior et al. (2007).

Foram observadas variações de 0 a $100 \%$ no grau de DE, indicando que existem projetos sem a presença de estrutura espacial e projetos onde toda a variação foi explicada pela componente espacial. Dos cinquenta projetos analisados, $64 \%$ apresentaram grau de dependência espacial (DE) de médio a forte. Situação semelhante foi encontrada por Kanegae Júnior et al. (2007), em estudo com plantios de eucalipto no estado de São Paulo com idade aproximada de 7 anos. Nos projetos com grau de dependência espacial de médio a forte, é possível gerar mapas que correspondem a uma pós-estratificação com base no volume, sem tendência e não enviesados, isto é, mais precisos no detalhamento espacial da variável dendrométrica de interesse (ASSIS, 2005).

\section{Estratificação com base nos interpoladores espaciais}

A Figura 2 apresenta o mapa de pósestratificação para o projeto $\mathrm{M} 3$, situado na região de Montes Claros. Este projeto apresentou grau

TABELA 1: Volume médio ( $\mathrm{m}^{3} / \mathrm{ha}$ ) e coeficiente de variação (CV\%) para cada região avaliada.

TABLE 1: Mean volume $\left(\mathrm{m}^{3} / \mathrm{ha}\right)$ and coefficient of variation $(\mathrm{CV} \%)$ for each region evaluated.

\begin{tabular}{c|c|c|c}
\hline \multicolumn{3}{c}{ Região } \\
\hline Idade & Curvelo & João Pinheiro & Montes Claros \\
\hline 24 & $37,11(34,59 \%)$ & $28,70(22,05 \%)$ & $32,24(33,91 \%)$ \\
36 & $143,25(18,26 \%)$ & $121,52(15,38 \%)$ & $107,53(16,99 \%)$ \\
48 & $168,43(18,77 \%)$ & $139,70(25,82 \%)$ & $139,30(18,35 \%)$ \\
60 & $206,95(11,06 \%)$ & $139,44(24,00 \%)$ & $83,86(30,03 \%)$ \\
\hline
\end{tabular}



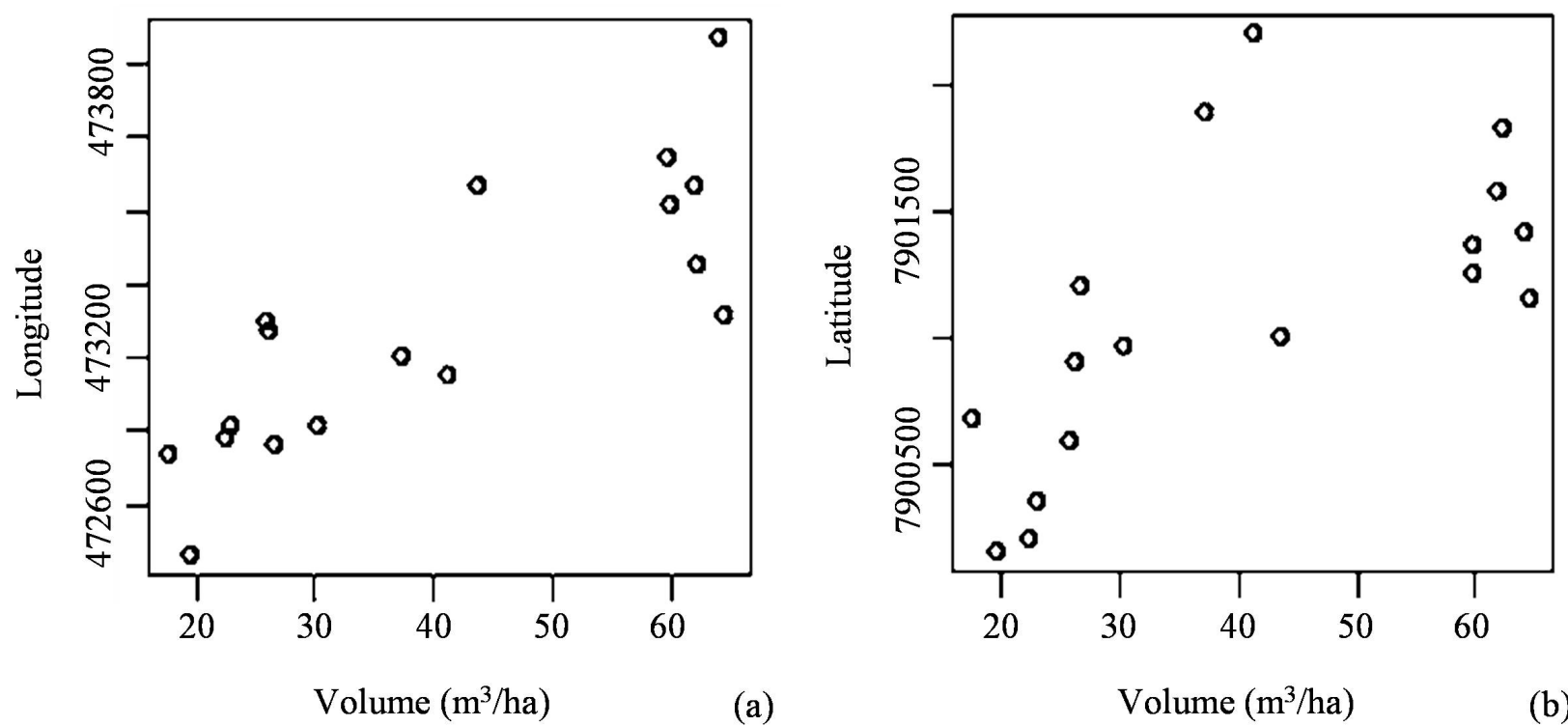

FIGURA 1: Relação entre o volume com a longitude (a) e com a latitude (b) para o projeto C12. FIGURE 1: Relationship between volume with longitude (a) and latitude (b) for C12 project.

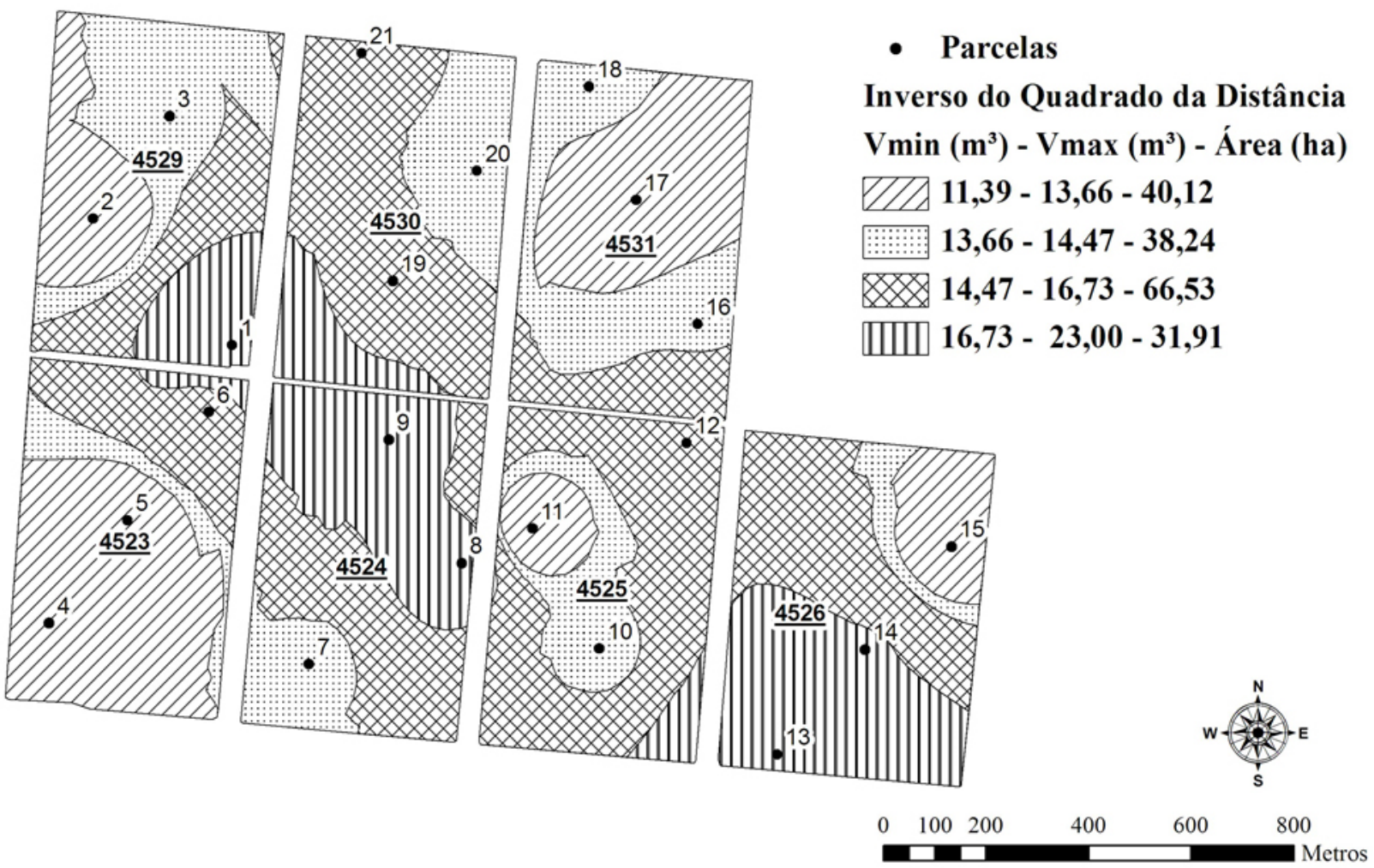

FIGURA 2: Estratificação obtida por meio do IQD para o projeto M3.

FIGURE 2: Stratification obtained by IQD for the M3 project.

de dependência espacial (DE) fraco, conforme a classificação proposta por Biondi et al. (1994). As Figuras 3 e 4 mostram os mapas de pósestratificação gerados para os projetos C11, situado no município de Curvelo, que apresentou grau de DE médio e para o projeto J2, situado no município de João Pinheiro, que apresentou forte grau de DE. Em termos espaciais, o IQD e a KRG definiram as mesmas regiões de produtividade. No entanto, a quantificação de área (em hectares) variou de estrato para estrato entre os dois interpoladores. 

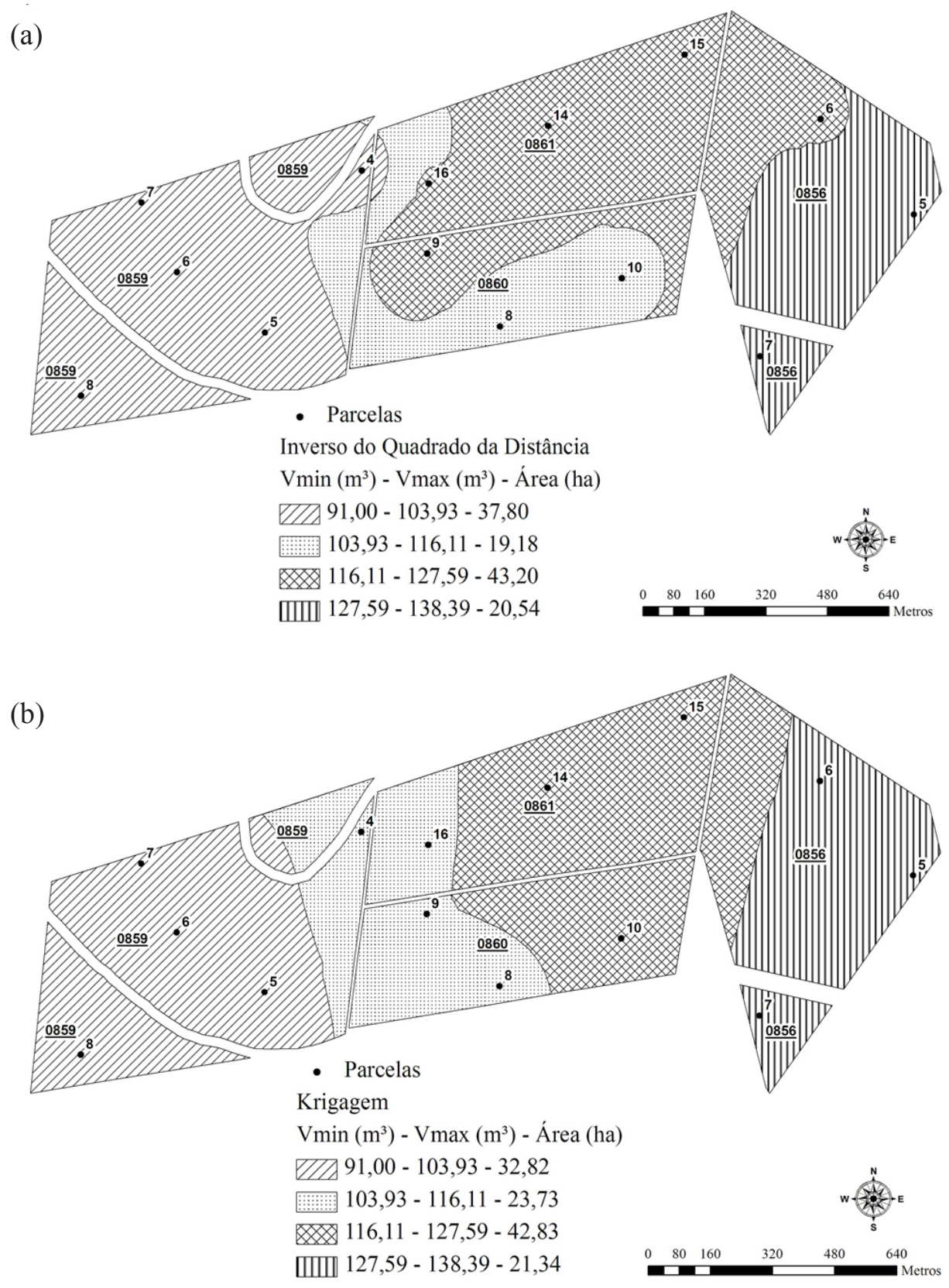

FIGURA 3: Estratificação obtida por meio do IQD (a) e da Krigagem (b) para o projeto C11. FIGURE 3: Stratification obtained by IQD (a) and Kriging (b) for the C11 project.

Avaliando os mapas dos projetos onde a pós-estratificação foi realizada por meio dos dois interpoladores (Figuras 3 e 4), percebe-se que o IQD superestimou a classe de maior volume. Resultados semelhantes foram encontrados por Kanegae Júnior (2004) em estudos de dependência espacial de Eucalyptus sp. em três regiões do estado de São Paulo. A alteração na área do estrato, afeta a proporção de cada estrato em relação à área total. Esta proporção participa dos cálculos de média e variância. Portanto, as estimativas serão diferentes quando comparadas às estatísticas obtidas pelos dois meios de estratificação. A diferenciação das áreas entre os estratos pode ter sido provocada devido ao fato de que o interpolador IQD pode estar viciado, uma vez que a soma dos pesos não é necessariamente igual a um, enquanto que a krigagem consiste de um interpolador exato e sem viés, onde a soma dos pesos é sempre igual a 1. Além disto, o interpolador IQD não considera a redundância de informações de 
(a)

- Parcelas

Inverso do Quadrado da Distância

$\operatorname{Vmin}\left(\mathrm{m}^{3}\right)$ - Vmax $\left(\mathrm{m}^{3}\right)$ - Área (ha)

VIIA 8,39 - 18,52 - 39,80

$18,52-26,02-98,11$

26 26,02 - 31,58 - 42,68

III] 31,58 - 35,70 - 36,57

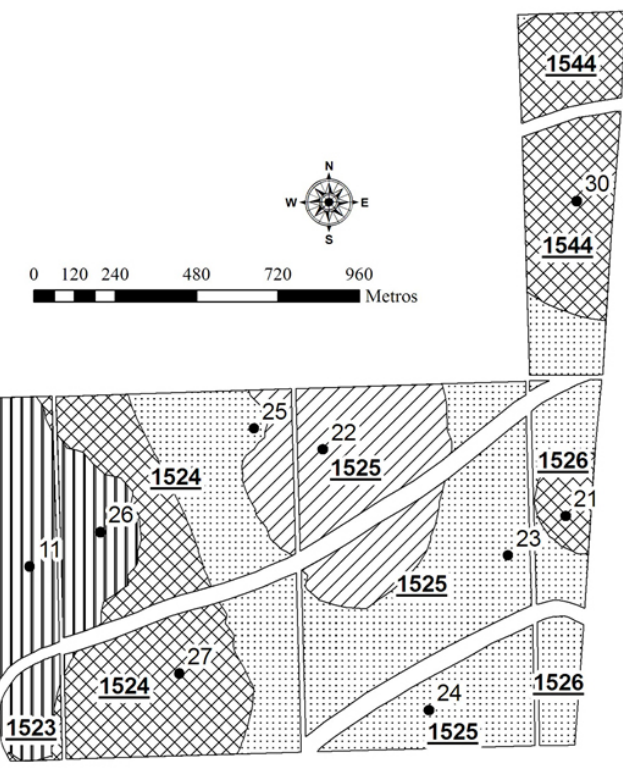

(b)

- Parcelas

Krigagem

$\operatorname{Vmin}\left(\mathrm{m}^{3}\right)$ - Vmax $\left(\mathrm{m}^{3}\right)$ - Área (ha)

VIT 8,39 - 18,52 - 52,73

$18,52-26,02-83,25$

26,02 - 31,58 - 46,83

|III 31,58 - 35,70 - 34,35
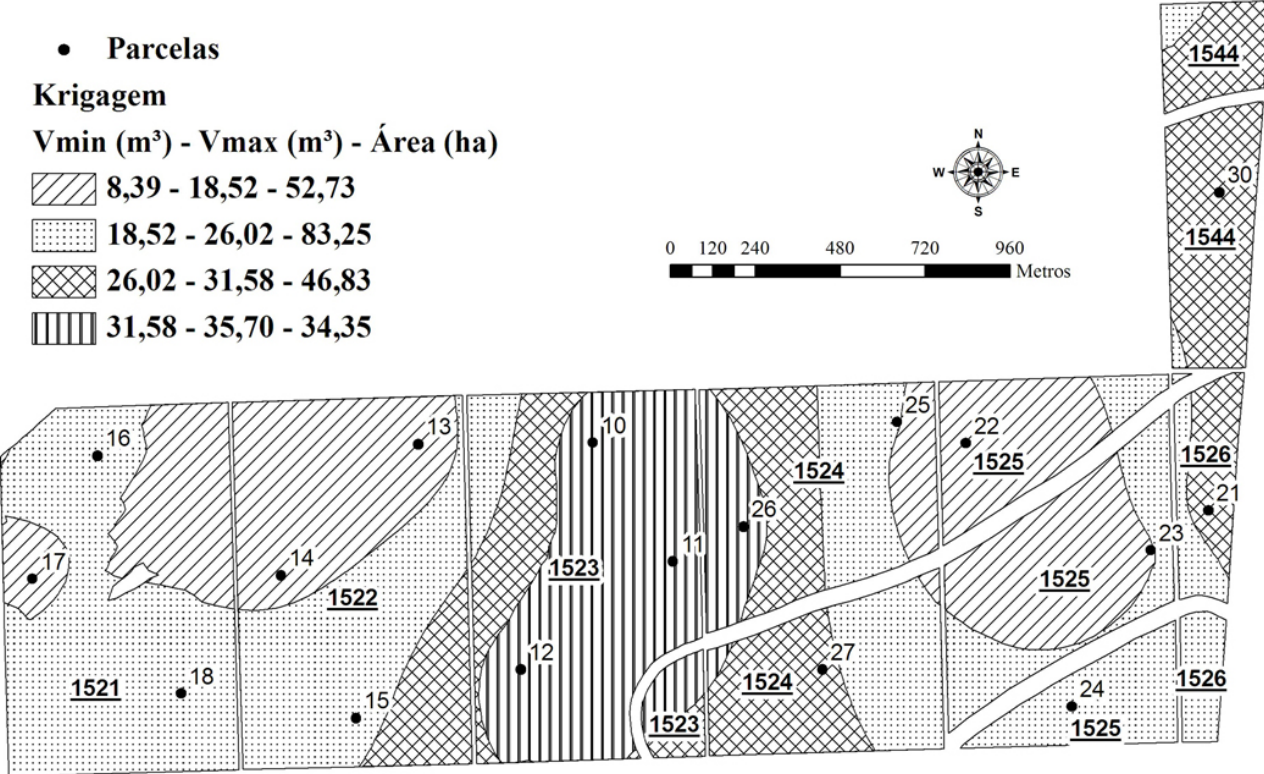

FIGURA 4: Estratificação obtida por meio do IQD (a) e da Krigagem (b) para o projeto J2. FIGURE 4: Stratification obtained by IQD (a) and Kriging (b) for the J2 project.

parcelas próximas e na krigagem, o peso de parcelas próximas, é muito pequeno na estimativa de pontos desconhecidos, propiciando economia de amostras sem comprometimento da precisão.

\section{Processamento do inventário florestal}

Na Tabela 2 tem-se os resultados de volume médio $\left(\mathrm{m}^{3} / \mathrm{ha}\right)$, erro padrão da média $\left(\mathrm{m}^{3} / \mathrm{ha}\right)$ e coeficiente de variação $(\mathrm{CV} \%)$ para os projetos que apresentaram fraca, média e forte dependência espacial (DE), para os diferentes procedimentos de amostragem considerados. É possível perceber que a estratificação contribuiu para uma redução média na variabilidade $(\mathrm{CV} \%)$ da ordem de $69,4 \%$ para os projetos com fraca DE, de $49,0 \%$ e $67,8 \%$ para os projetos que apresentaram média e forte $\mathrm{DE}$, respectivamente.

Kanegae Júnior et al. (2006) verificou redução de 66 \% na variabilidade, quando efetuou a pós-estratificação com base no volume de eucalipto por meio da krigagem. Estes resultados salientam a importância da pós-estratificação com base na característica de interesse, que permite o aumento da precisão sem causar impactos no custo do inventário. 
TABELA 2: Volume médio, desvio padrão da média e coeficiente de variação para projetos em diferentes idades e regiões.

TABLE 2: Mean volume, mean standard error and coefficient of variation for projects in different ages and regions.

\begin{tabular}{|c|c|c|c|c|c|c|c|c|c|c|c|}
\hline & & & & ACS & & & CE (IQD) & & & E (KRG & \\
\hline $\mathrm{DE}$ & Projeto $^{1}$ & Idade & $\begin{array}{c}\bar{V} \\
\left(\mathrm{~m}^{3} / \mathrm{ha}\right)\end{array}$ & $\begin{array}{c}S_{\bar{V}} \\
\left(\mathrm{~m}^{3} / \mathrm{ha}\right)\end{array}$ & $\begin{array}{l}\text { CV } \\
(\%)\end{array}$ & $\begin{array}{c}\bar{V}_{s t r} \\
\left(\mathrm{~m}^{3} / \mathrm{ha}\right)\end{array}$ & $\begin{array}{c}S_{\overline{\bar{V}}_{s t r}} \\
\left(\mathrm{~m}^{3} / \mathrm{ha}\right)\end{array}$ & $\begin{array}{l}\text { CV } \\
(\%)\end{array}$ & $\begin{array}{c}\bar{V}_{s t r} \\
\left(\mathrm{~m}^{3} / \mathrm{ha}\right)\end{array}$ & $\begin{array}{c}S_{\overline{V_{s}} r} \\
\left(\mathrm{~m}^{3} / \mathrm{ha}\right)\end{array}$ & $\begin{array}{l}\text { CV } \\
(\%)\end{array}$ \\
\hline & $\mathrm{J} 1$ & 24 & 23,1 & 0,7947 & 21,27 & 23,09 & 0,21 & 5,8 & - & - & - \\
\hline 넥 & M3 & 24 & 15,1 & 0,7277 & 22,11 & 15,12 & 0,28 & 8,71 & - & - & - \\
\hline త్ర & $\mathrm{J} 8$ & 36 & 136,7 & 6,1517 & 18,03 & 136,34 & 1,77 & 4,99 & - & - & - \\
\hline 部 & C13 & 48 & 152,6 & 5,7491 & 15,55 & 156,26 & 1,72 & 4,34 & - & - & - \\
\hline & $\mathrm{C} 18$ & 48 & 172,9 & 5,9936 & 15,52 & 166,61 & 1,98 & 4,81 & - & - & - \\
\hline & $\mathrm{J} 4$ & 24 & 27,4 & 1,12 & 18,35 & 27,2 & 0,34 & 5,72 & 26,6 & 0,52 & 8,12 \\
\hline 屸 & $\mathrm{C} 2$ & 24 & 59,8 & 2,27 & 13,7 & 60,55 & 0,76 & 4,42 & 61,22 & 1,34 & 7,46 \\
\hline$\stackrel{\frac{\pi}{7}}{7}$ & $\mathrm{~J} 4$ & 24 & 154,6 & 4,41 & 18,74 & 155,7 & 1,73 & 7,37 & 157,4 & 2,76 & 11,21 \\
\hline$\sum$ & C11 & 36 & 114,3 & 4,5 & 14,73 & 115,7 & 1,22 & 4,02 & 115 & 2,34 & 7,71 \\
\hline & $\mathrm{J} 12$ & 48 & 113,2 & 5,72 & 37,21 & 115,5 & 1,91 & 12,05 & 113,9 & 2,65 & 16,44 \\
\hline & $\mathrm{J} 2$ & 24 & 23,8 & 1,96 & 33,94 & 23,4 & 0,66 & 11,54 & 23,5 & 0,63 & 11,13 \\
\hline 닉 & M9 & 36 & 132,7 & 6,65 & 22,98 & 135,3 & 1,75 & 5,61 & 131,6 & 2,36 & 6,62 \\
\hline$\stackrel{0}{0}$ & J13 & 48 & 156,2 & 7,53 & 19,89 & 157,8 & 2,03 & 5,31 & 156,4 & 2,4 & 5,65 \\
\hline î & $\mathrm{C} 17$ & 48 & 121,4 & 11,76 & 38,77 & 125,5 & 2,97 & 9,27 & 124,1 & 4,04 & 12,81 \\
\hline & M14 & 60 & 100,9 & 6,31 & 33,17 & 100,5 & 1,92 & 10,32 & 99,1 & 2,32 & 12,54 \\
\hline
\end{tabular}

Em que: ${ }^{1} \mathrm{~J}$ : João Pinheiro, M: Montes Claros e C: Curvelo.

É possível também afirmar que, para futuras amostragens, pode-se utilizar um número menor de parcelas para se obter um erro desejável para o levantamento, com redução de custo envolvido no processo, pois, o custo do inventário é diretamente influenciado pelo tempo de medição (CESARO et al., 1994). Estes resultados demonstram que tanto o método geoestatístico quanto outro interpolador espacial, reduz custos no inventário florestal, fazendo uso dos estimadores da amostragem casual estratificada.

Na Tabela 3 apresenta-se o erro do inventário, em porcentagem, para os quinze projetos selecionados para a realização da estratificação. Percebe-se que o procedimento da Amostragem Casual Estratificada foi superior ao da Amostragem Casual Simples, independente do interpolador utilizado. Desta forma, pode-se afirmar que, mesmo que o analista não saiba detectar a existência da dependência espacial, a estratificação com base no interpolador IQD, que é viciado quando comparado com a Krigagem, é uma alternativa interessante para aumentar a precisão das estimativas (ASSIS, 2005, KANEGAE JÚNIOR et al., 2006).

A estratificação com base no interpolador IQD utilizado nos projetos que apresentaram fraca dependência espacial contribuiu para uma redução em média de $68,4 \%$ no erro de amostragem. A estratificação com base no interpolador geoestatístico naqueles projetos que apresentaram grau de estrutura de continuidade espacial médio e forte, contribuiu para uma redução média de $47,0 \%$ e $65,7 \%$ no erro de amostragem, respectivamente. Resultados semelhantes foram encontrados por Kanegae Júnior et al. (2006), que utilizaram o interpolador IQD com base na característica volume para a realização da estratificação e constataram uma redução média no erro de amostragem de $48 \%$ em relação à Amostragem Casual Simples. Assis (2005) também obteve resultados semelhantes, tendo encontrado uma redução média do erro padrão da média de $61,7 \%$ quando comparou a ACS com a ACE, com a estratificação realizada através da krigagem, com base na característica volume. 
TABELA 3: Erro do inventário (\%), considerando a Amostragem Casual Simples (ACS), Amostragem Casual Estratificada com estratos obtidos por meio do IQD (ACE IQD) e Krigagem (ACE KRG).

TABLE 3: Inventory error (\%), considering the Random Sampling Design (ACS), Stratified Sampling Design with stratus obtained by IQD (ACE IQD) and Kriging (ACE KRG).

\begin{tabular}{|c|c|c|c|c|c|}
\hline $\mathrm{DE}$ & Projeto & IDADE & ACS & $\begin{array}{c}\text { ACE } \\
\text { (IQD) }\end{array}$ & $\begin{array}{c}\mathrm{ACE} \\
(\mathrm{KRG})\end{array}$ \\
\hline \multirow{5}{*}{ 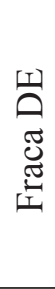 } & $\mathrm{J} 1$ & 24 & 6,98 & 1,86 & - \\
\hline & M3 & 24 & 10,05 & 3,90 & - \\
\hline & $\mathrm{J} 8$ & 36 & 9,60 & 2,77 & - \\
\hline & $\mathrm{C} 13$ & 48 & 7,99 & 2,34 & - \\
\hline & $\mathrm{C} 18$ & 48 & 7,26 & 2,49 & - \\
\hline \multirow{5}{*}{ 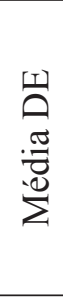 } & $\mathrm{J} 4$ & 24 & 8,58 & 2,58 & 4,09 \\
\hline & $\mathrm{C} 2$ & 24 & 8,27 & 4,81 & 4,79 \\
\hline & $\mathrm{C} 11$ & 36 & 8,50 & 2,28 & 4,40 \\
\hline & $\mathrm{J} 12$ & 48 & 10,13 & 3,32 & 4,67 \\
\hline & $\mathrm{J} 14$ & 48 & 5,76 & 2,25 & 3,54 \\
\hline \multirow{5}{*}{$\begin{array}{l}\text { II } \\
0 \\
0 \\
0 \\
0 \\
0\end{array}$} & $\mathrm{~J} 2$ & 24 & 17,43 & 5,96 & 5,70 \\
\hline & M9 & 36 & 10,45 & 2,71 & 3,74 \\
\hline & $\mathrm{J} 13$ & 48 & 10,22 & 2,72 & 3,25 \\
\hline & $\mathrm{C} 17$ & 48 & 20,64 & 5,04 & 6,93 \\
\hline & M14 & 60 & 12,84 & 3,92 & 4,80 \\
\hline
\end{tabular}

Portanto, o uso de interpoladores espaciais permite gerar estratos, através dos quais, conseguese uma redução significativa no erro do inventário, sem aumentar o custo para o levantamento. Se houver dependência espacial superior a $35 \%$, a krigagem deve ser escolhida. No entanto, na ausência de dependência espacial, ou mesmo na presença de fraca estrutura de dependência, o uso do IQD pode ser pertinente para obter a pós-estratificação com base na característica de interesse. Assim como afirmaram Kanegae Júnior et al. (2006) e Assis (2005), a técnica de pós-estratificação deve ser sempre considerada no intuito de reduzir o erro do inventário, e consequentemente seus custos.

\section{CONCLUSÕES}

Dos cinquenta projetos analisados, $64 \%$ apresentaram grau de dependência espacial (DE) de médio a forte, evidenciando assim que o uso de métodos geoestatísticos possibilita redução no erro sem aumento de custo no inventário, por meio da estratificação utilizando a krigagem.

Nos projetos que apresentaram fraca DE a estratificação com base no interpolador inverso do quadrado das distâncias (IQD) contribuiu para uma redução em média de 68,4 \% no erro de amostragem.

Nos projetos que apresentaram média e forte DE a estratificação com base no interpolador geoestatístico contribuiu para uma redução média de $47,0 \%$ e $65,7 \%$ no erro de amostragem, respectivamente.

A estratificação com base no interpolador IQD gera melhoria das estimativas da ACE em relação à ACS mesmo nos projetos onde a estrutura de dependência espacial se manifestou.

Os métodos dos interpoladores espaciais têm potencial para uso na definição de estratos em povoamentos de Eucalyptus sp., uma vez que se mostraram eficientes para a definição de estratos com base na característica de interesse, gerando ganhos de precisão para as estimativas de volume.

\section{REFERÊNCIAS BIBLIOGRÁFICAS}

ANTUNES, F. Z. Caracterização climática do Estado de Minas Gerais. Informe Agropecuário, Belo Horizonte, v. 12, n. 138, p. 9-13, jan. 1986. ASSIS, A. L. de. Definição da estratégia amostral em plantios jovens de Eucalyptus spp pelo uso de geoestatística. 2005. 118 p. Tese (Doutorado em Engenharia Florestal)-Universidade Federal de Lavras, Lavras, 2005.

BIONDI, F. et al. Geostatistically modeling stem size and increment in an old-growth forest. Canadian Journal of Forest Research-Revue Canadienne de Recherche Forestiere, Ottawa, v. 24, n. 7, p.1354-1368, July 1994.

BOYDEN, S. et al. Competition among Eucalyptus trees depends on genetic variation and resource supply. Ecology, v. 89, n. 10, p. 2850-2859, 2008.

CESARO, A. de. et al. Comparação dos métodos de amostragem de área fixa, relascopia, e de seis árvores, quanto a eficiência, no inventario florestal de um povoamento de Pinus sp. Ciência Florestal, Santa Maria, v. 4, n. 1, p. 97-108, jun 1994.

COCHRAN, W. G. Sampling techniques. 3rd ed. New York: J. Wiley, 1977. 555 p.

CRESSIE, A. G. Statistics for spatial data. New York: J. Wiley, 1993.900 p.

DIGGLE, P. J.; RIBEIRO JÚNIOR, P. J. Model 
based geostatistics. Londres: Springer, 2007. 230 p.

FERREIRA, D. F. Estatística básica. Lavras: UFLA, 2005. 664 p.

ISAAKS, E. H.; SRIVASTAVA, R. M. An introduction to applied geostatistics. New York: Oxford University, 1989. $560 \mathrm{p}$.

JOURnEL, A. G.; HUIJBREGTS, C. J. Mining geostatistics. London: Academic, 1978. 600 p.

KANEGAE JÚNIOR, $H$. et al. Avaliação da continuidade espacial de características dendrométricas em diferentes idades de povoamentos clonais de Eucalyptus sp. Revista Árvore, Viçosa, v. 31, n. 5, p. 859-866, set./out. 2007.

KANEGAE JÚNIOR, $H$. et al. Avaliação de interpoladores estatísticos e determinísticos como instrumento de estratificação de povoamentos clonais de Eucalyptus sp. Cerne, Lavras, v. 12, n. 2, p. 123-136, abr./jun. 2006

LANDIM, P. M. B. Análise estatística de dados geológicos. São Paulo:UNESP, 1998. 226 p.

MACHADO, S. A. et al. Comparação entre métodos para avaliação de volume total de madeira por unidade de área, para o pinheiro do Paraná, na região sul do Brasil. Cerne, Lavras, v. 6, n. 2, p. 55-66, 2000.

MATHERON, G. Principles of geostatistics. Economic geology, Littleton, v. 58, p. 1246-1266, 1963.

MELLO, J. M. de. et al. Ajuste e seleção de modelos espaciais de semivariograma visando à estimativa volumétrica de Eucalyptus grandis. Scientia Forestalis, Piracicaba, n. 69, p. 25-37, dez. 2005b. MELLO, J. M. de. et al. Continuidade espacial para características dendrométricas (número de fustes e volume) em plantios de Eucalyptus grandis. Revista Árvore, Viçosa, v. 33, n. 1, jan./fev., 2009. MELLO, J. M. de. et al. Estudo da dependência espacial de características dendrométricas para Eucalyptus grandis. Cerne, Lavras, v. 11, n. 2, p. 113-126, abr./jun. 2005a.
MELLO, J. M. de.; SCOLFORO, J. R. S. Análise comparativa de procedimentos de amostragem em um remanescente de floresta estacional semidecídua montana. Revista Árvore, Viçosa, v. 24, n. 1, p. 55-62, 2000.

MENG, Q. et al. Large area forest inventory using Landsat ETM+: a geostatistical approach. ISPRS Journal of Photogrammetry and Remote Sensing, v.64, $\mathrm{n}$.

PÉLLICO NETTO, S.; BRENA, D. A. Inventário florestal. Curitiba: [s.n.], 1997. 316 p.

PÉlLICO NETTO, S.; SANQUETTA, C. R. Determinação do número de estratos em estratificação volumétrica de florestas naturais e plantadas. Floresta, Curitiba, v. 24, n. 1-2, p.4958, jun./dez. 1994.

PRODAN, M. et al. Mensura forestal. São José, Costa Rica: IICA/BMZ/GTZ, 1997. 586 p.

R DEVELOPMENT CORE TEAM. R: a language and environment for statistical computing. Vienna: R Foundation for Statistical Computing. 2007. Disponível em: < http://www.r-project.org $>$.

RIBEIRO JÚNIOR, P. J.; DIGGLE, P. J. geoR: a package for geostatistical analysis. R-NEWS, Pelotas, v.1, n.2, p.15-18, 2001.

SCOLFORO, J. R. S.; MELLO, J. M. de. Inventário florestal. Lavras: UFLA/FAEPE, 2006. 561 p.

SHAPIRO, S. S.; WILK, M. B. An analysis of variance test for normality (complete samples). Biometrika, London, v. 53, n. 3/4, p. 591-611, July/Dec. 1965.

VIEIRA, S. R. Geoestatística em estudos de variabilidade espacial do solo. In: NOVAIS, R. F.; ALVAREZ, V. H.; SCHAEFER, C. E. G. R. (Ed.). Tópicos em ciências do solo. Viçosa: SBCS, 2000. WEBSTER, R; McBRATNEY, A. B. On the Akaike Information Criterion for choosing models for variograms of soil properties. European Journal of Soil Science, Oxford, v. 40, n. 3, p. 493-496, Sept. 1989.

YAMAMOTO, J. K. Avaliação e classificação de reservas minerais. São Paulo: USP, 2001. 226 p. 